\title{
Defining the relationship of the optic radiation to the roof and floor of the ventricular atrium: a focused microanatomical study
}

\author{
Christos Koutsarnakis, MD,, Aristotelis V. Kalyvas, MD, MSc, ${ }^{1,2}$ Spyridon Komaitis, MD, ${ }^{1,2}$ \\ Faidon Liakos, MD, ${ }^{1}$ Georgios P. Skandalakis, BBA, MSc, ${ }^{1,3,5}$ Christos Anagnostopoulos, MD, ${ }^{2}$ and \\ George Stranjalis, MD ${ }^{1-5}$
}

\begin{abstract}
${ }^{1}$ Athens Microneurosurgery Laboratory, Evangelismos Hospital; '2Department of Neurosurgery, Evangelismos Hospital, and ${ }^{3}$ Department of Anatomy, ${ }^{4}$ Medical School, National and Kapodistrian University of Athens; and ${ }^{5}$ Hellenic Center for Neurosurgical Research "Petros Kokkalis," Athens, Greece
\end{abstract}

\begin{abstract}
OBJECTIVE The authors investigated the specific topographic relationship of the optic radiation fibers to the roof and floor of the ventricular atrium because the current literature is ambiguous.

METHODS Thirty-five normal, adult, formalin-fixed cerebral hemispheres and 30 focused MRI slices at the level of the atrium were included in the study. The correlative anatomy of the optic radiation with regard to the atrial roof and floor was investigated in 15 specimens, each through focused fiber microdissections. The remaining 5 hemispheres were explored with particular emphasis on the trajectory of the collateral sulcus in relation to the floor of the atrium. In addition, the trajectory of the collateral sulcus was evaluated in $30 \mathrm{MRI}$ scans.
\end{abstract}

RESULTS The atrial roof was observed to be devoid of optic radiations in all studied hemispheres, whereas the atrial floor was seen to harbor optic fibers on its lateral part. Moreover, the trajectory of the intraparietal sulcus, when followed, was always seen to correspond to the roof of the atrium, thus avoiding the optic pathway, whereas that of the collateral sulcus was found to lead to either the lateral atrial floor or outside the ventricle in $88 \%$ of the cases, therefore hitting the visual pathway.

CONCLUSIONS Operative corridors accessing the ventricular atrium should be carefully tailored through detailed preoperative planning and effective use of intraoperative navigation to increase patient safety and enhance the surgeon's maneuverability. The authors strongly emphasize the significance of accurate anatomical knowledge.

https://thejns.org/doi/abs/10.3171/2017.10.JNS171836

KEYWORDS optic radiations; collateral sulcus; intraparietal sulcus; ventricular atrium; ventricular approaches; white matter anatomy

$\mathrm{T}$ $\mathrm{HE}$ atrium of the lateral ventricle is a common yet very challenging site for surgical exploration because of its deep location, small size, and intricate anatomy. $1,27,31,33,39,50$ In particular, its inherent relationship to the fibers of the optic radiation has led to the recommendation for using different operative trajectories to enhance patient safety by minimizing risk of injury to the optic pathways while attempting to maximize the working corridor and the surgeon's maneuverability.,12,13,15, $17-19,27,29,32-34,43,49,50$
In this context, several studies have sought to investigate the topographic anatomy and architecture of the optic bundles with regard to the ventricular trigone and have consistently documented the lateral and medial atrial walls to be full and devoid of optic fibers, respectively. ${ }^{5,8,}$ 9,12,15,27,36,37,42-44 As a result, transventricular approaches to the atrium through its medial aspect preserve the visual pathways, whereas operative trajectories directed to the lateral atrial wall disrupt optic fibers and lead to postoperative visual deficit. 
Nonetheless, information in the current literature is vague and, at times, contradictory when the relationship of the optic radiations to the atrial roof and floor is concerned. Some authors have argued that part of the roof and floor of the ventricular trigone is covered by optic fibers, $34,36,37,42$ whereas others have claimed exactly the opposite. ${ }^{12,13,15}$ As a consequence, ambiguity remains as to whether surgical access to the atrial roof and floor through the most common superiorly or inferiorly directed transcortical or transsulcal transventricular variants, such as the superior parietal lobule, intraparietal sulcus, transcollateral sulcus, or subtemporal approach, confers risk of injury to the visual pathways.

Hence, our main objective was to shed light on this topic by exploring the topographic relationship of the optic radiation to the roof and floor of the ventricular atrium through focused white matter fiber microdissections. In addition, we discuss our findings in relation to some of the most commonly used transventricular approaches for the treatment of atrial lesions.

\section{Methods}

This study included 35 normal adult cadaveric cerebral hemispheres (19 right hemispheres, 16 left hemispheres) obtained from 35 cadavers previously fixed in a 10\%-15\% formalin solution for a minimum of 8 weeks. After the arachnoid membrane and vessels were removed, all hemispheres underwent the Klingler procedure (freeze-thaw process) and subsequently were investigated through the fiber microdissection technique. ${ }^{16,20}$

Because the roof of the atrium is located in the depth of the parietal lobe, we opted to perform stepwise lateromedial microanatomical dissections focused on this re- gion in 15 specimens. Special emphasis was placed on the white matter lying in or adjacent to the dissection plane defined by the intraparietal sulcus, because as previously shown, the trajectory of this sulcus points toward the roof of the atrium..$^{10,17,19,40,41} \mathrm{We}$ extended our dissection to the posterior part of the superior temporal gyrus and to the most anterior part of the superior middle and inferior occipital gyri to expose both the lateral wall and roof of the atrium and to delineate more accurately their respective fiber tract anatomy. Thus, the white matter pathways that overlie the roof of the atrium were studied thoroughly and illustrated vividly.

Fifteen specimens were explored through white matter fiber dissections focused on the inferomedial temporooccipital surface and with an inferior-to-superior direction, since the floor of the atrium resides in the depth of the posteromedial temporal lobe and more particularly in the posterior segment of the fusiform gyrus. ${ }^{39-41,50}$ Therefore, we focused our dissections on the area of the parahippocampal and fusiform gyri with an extension to the lingual gyrus, isthmus of the cingulate gyrus, and part of the medial occipital surface known as the cuneus to explore and delineate the subcortical anatomy in relation to the part of the ventricle defined as the atrium. In this way, the topographic anatomy of the white matter pathways, and of the optic radiation to the floor of the atrium in particular, was investigated meticulously.

In the remaining 5 hemispheres, we specifically focused our dissection on the collateral sulcus, i.e., the sulcus demarcating the parahippocampal and lingual gyri from the fusiform gyrus, ${ }^{23,40,41}$ with the aim of defining the relationship of the surface anatomy and topography of this sulcus to the floor of the atrium.

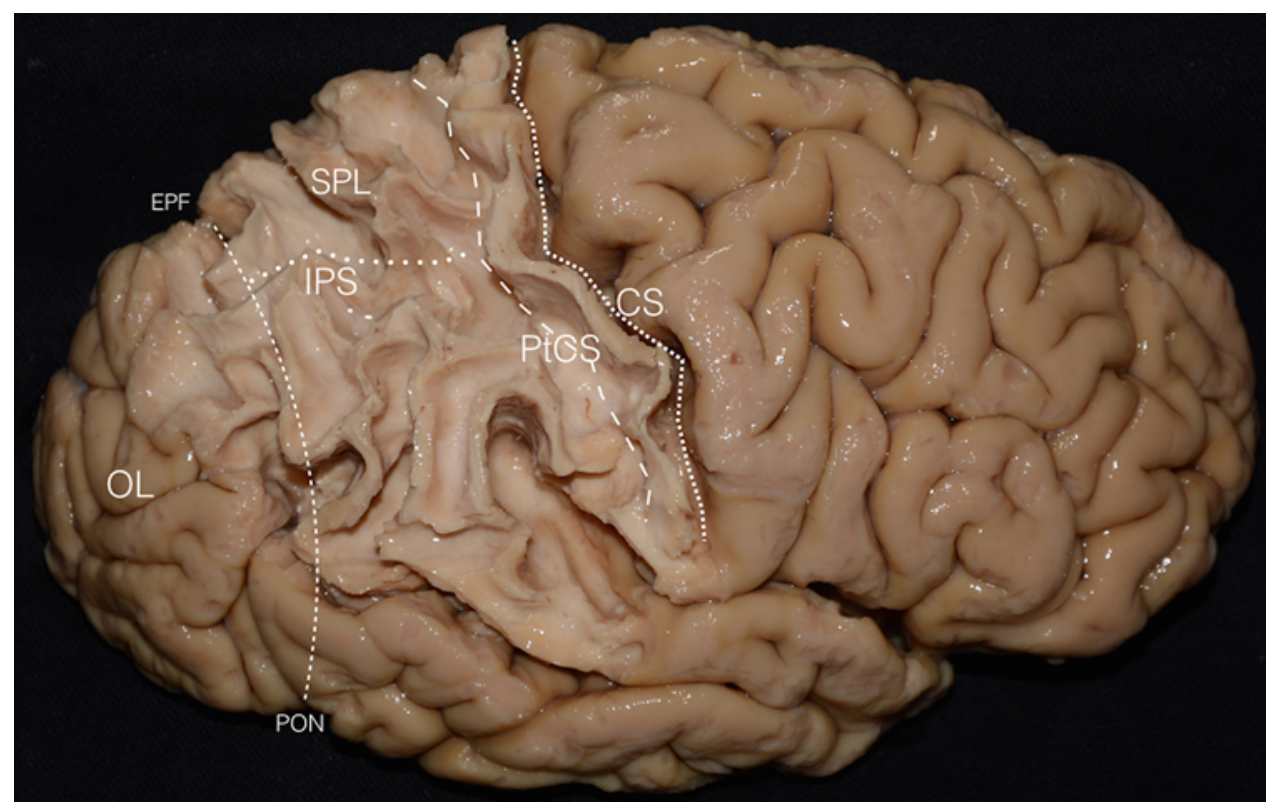

FIG. 1. Photograph of the lateral surface of a right cerebral hemisphere. The small arcuate (U) fibers of the parietal lobe are depicted. The level of the intraparietal sulcus, central sulcus, and postcentral sulcus is marked along with the boundaries of the occipital lobe. $C S$ = central sulcus; $E P F=$ external perpendicular fissure; IPS = intraparietal sulcus; OL = occipital lobe; $P O N=$ preoccipital notch; PtCS = postcentral sulcus; SPL = superior parietal lobule. Figure is available in color online only. 


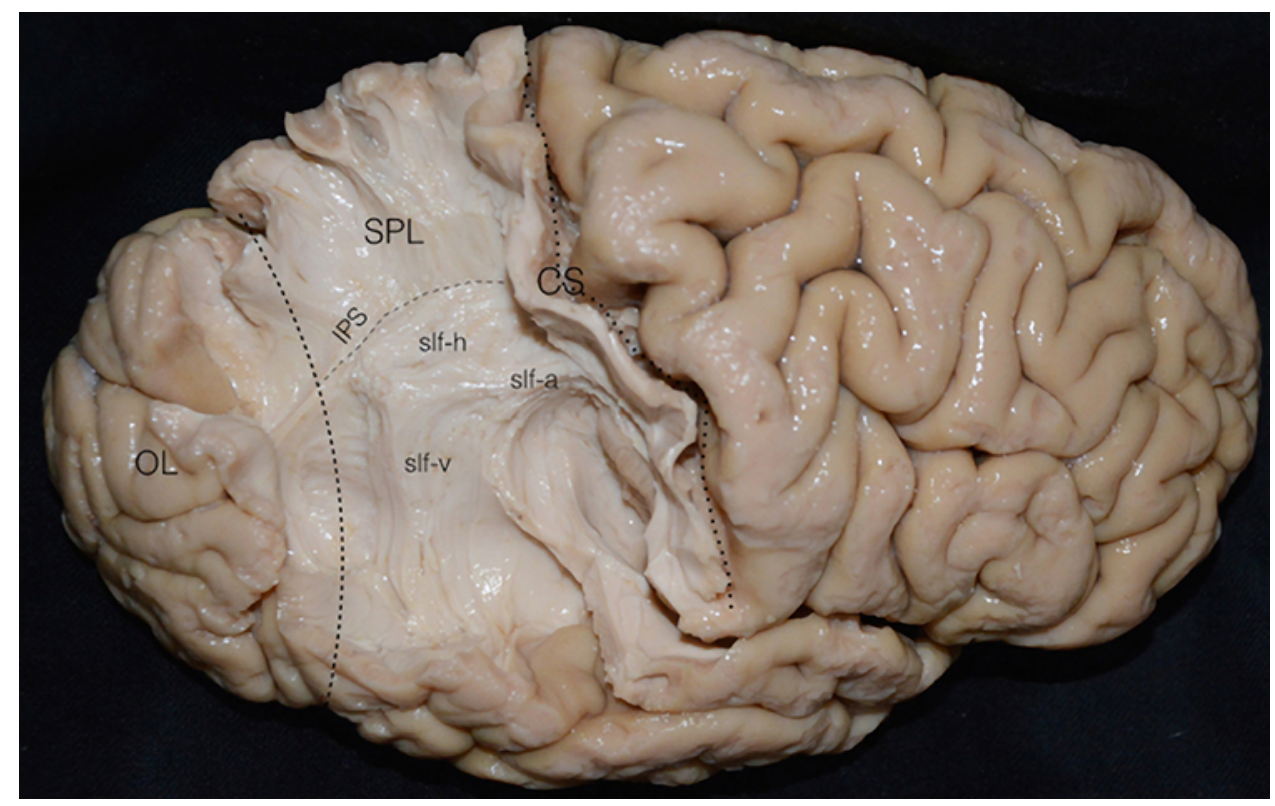

FIG. 2. Photograph showing dissection of the small arcuate $(U)$ fibers of the parietal area and posterior part of the superior temporal gyrus, which reveals the horizontal, vertical, and arcuate segments of the SLF. The plane of the intraparietal sulcus and the boundaries of the occipital lobe are marked with dotted lines. Note the tight anatomical relationship between the plane of the intraparietal sulcus and the SLF. slf-a = arcuate segment; slf- $h=$ horizontal segment; slf-v = vertical segment. Figure is available in color online only.

It has to be emphasized that at the level of the ventricular trigone, i.e., the posterior temporal and parietal areas, the fibers of the optic radiations intermingle tightly with several white matter bundles to form what is anatomically defined as the sagittal stratum. Because these converging fiber tracts share the same direction, orientation, and trajectory, it is impossible to dissect them away and isolate them. , $8,18,20,45,46$ Hence, at this level, and particularly in relation to the floor and roof of the atrium, the fibers of the sagittal stratum set the boundaries of the anatomical loca-

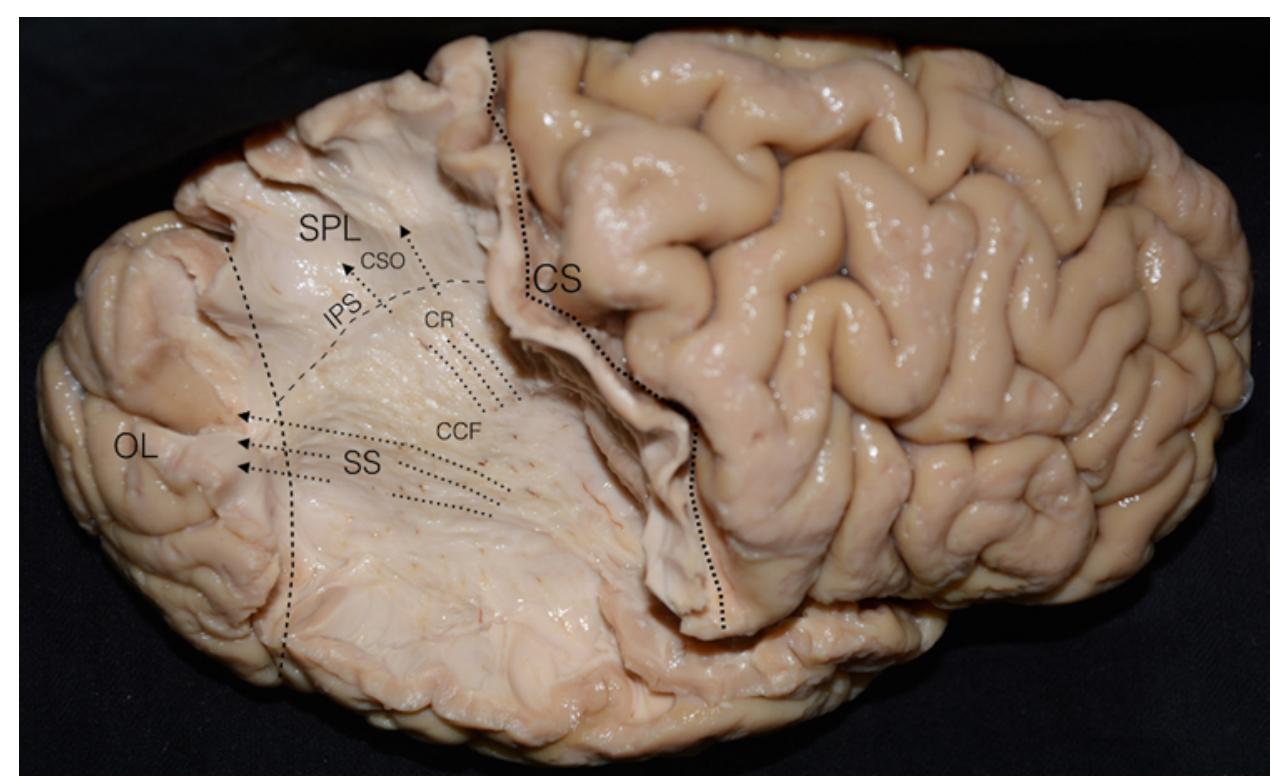

FIG. 3. Photograph showing progressive dissection of the specimen. The most laterally situated fibers of the corona radiata and sagittal stratum become apparent after removing the SLF. Note that the trajectory of the fibers of the sagittal stratum is toward the occipital lobe, whereas that of the corona radiata is toward the parietal lobe. These fibers are claustrocortical radiation fibers that form the external capsule as they advance in the area of the central core. The plane of the intraparietal sulcus marks a rough boundary between the corona radiata and the centrum semiovale, because this sulcus corresponds to the roof of the ventricular trigone. $\mathrm{CCF}=$ claustrocortical radiation fibers; $\mathrm{CR}=$ corona radiata; $\mathrm{CSO}=$ centrum semiovale; $\mathrm{SS}=$ sagittal stratum. Figure is available in color online only. 


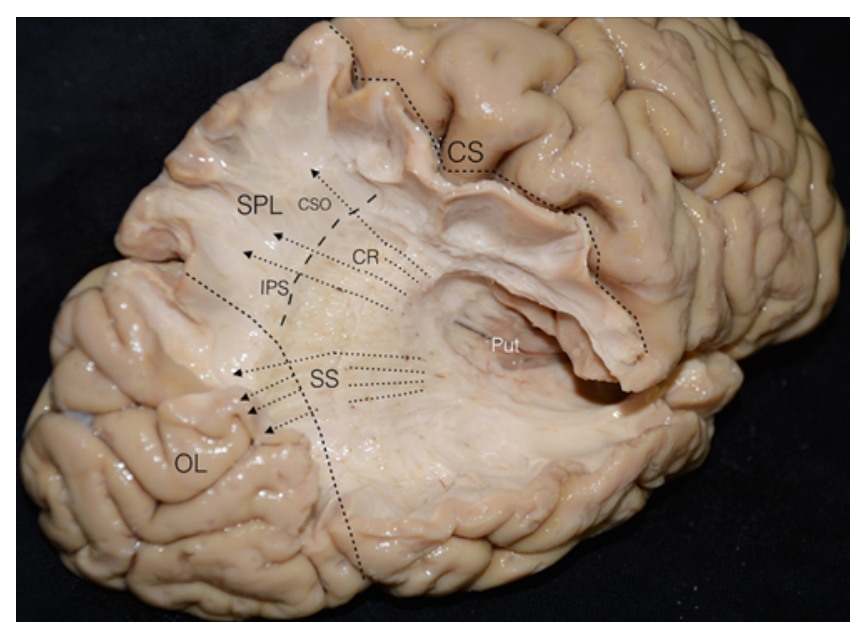

FIG. 4. Oblique photograph of the hemisphere after dissection of the claustrocortical fibers. The fibers of the internal capsule become apparent, forming the consecutive white matter layer of the corona radiata and sagittal stratum, along with the gray matter of the putamen. At this stage, these are fibers of the posterior and retrolenticular limbs of the internal capsule that fan out toward the parietal and occipital lobes. Note that the fibers of the internal capsule lying deep to the plane of the intraparietal sulcus, and thus covering the roof of the atrium, radiate consistently toward the white matter of the superior parietal lobule, forming a part of the corona radiata and centrum semiovale. The fibers of the sagittal stratum, in contrast, radiate toward the occipital lobe, as clearly illustrated. No fibers were seen to travel all the way up to the plane of the intraparietal sulcus and then bend toward the occipital lobe, thus giving the impression of the optic radiation. Put = putamen. Figure is available in color online only.

tion of the visual pathway, because the latter structure is included in the former one.

We also explored the correlation between the trajectory of the collateral sulcus and the floor of the atrium in 30 focused coronal MRI slices of 30 individuals to evaluate the anatomy of 60 sulci. Informed consent was obtained from all patients before the inclusion of their scans in the study.

The dissection tools used were fine metallic periosteal elevators, variously sized anatomical forceps, and microsurgical scissors, because they were easier to use and more precise in their handling than wooden spatulas. ${ }^{20-22}$ During the dissections, numerous photographs were acquired to illustrate the regional cortical and subcortical anatomies of interest vividly. It must be highlighted that no photographs included in this study were edited by any image-correcting software so that they closely resemble the anatomy encountered during standard fiber microdissections in the setting of a microneurosurgical laboratory. ${ }^{19-22}$

\section{Results}

\section{Atrial Roof and Optic Radiations}

We applied the fiber microdissection technique in a stepwise fashion while remaining focused on the parietal lobe with the aim of investigating and defining the potential relationship between the atrial roof and the fibers of the visual pathway. During the dissection process, the plane of the intraparietal sulcus and the boundaries between the parietal and occipital lobes were demarcated
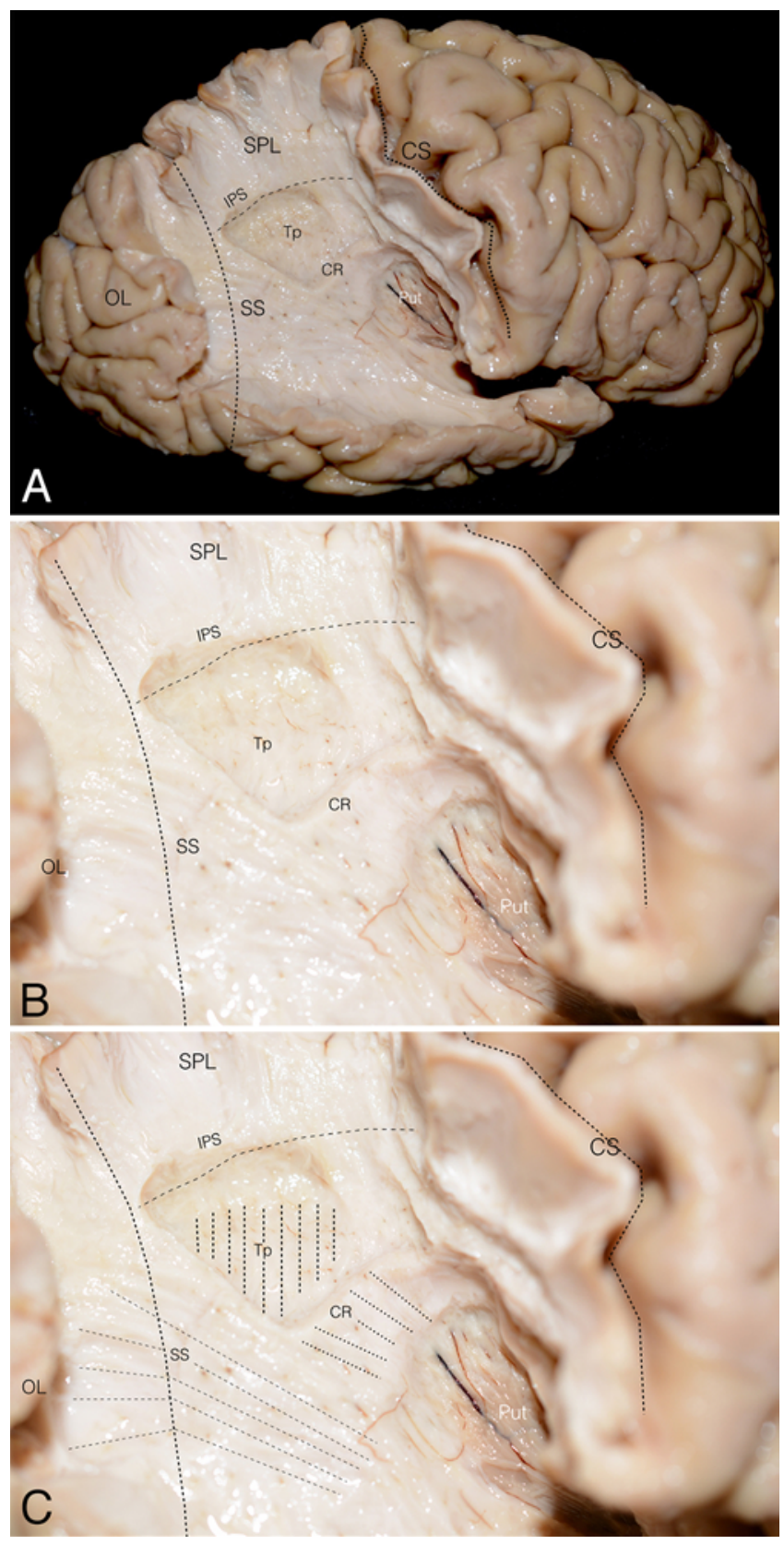

FIG. 5. A: Photograph of the specimen with focused dissection of the fibers of the internal capsule at the level of the roof of the atrium. The fibers of the tapetum become apparent with their characteristic trajectory, arching over the roof of the atrium. B and C: Magnified photographs of the regional anatomy, depicting the trajectory of the fibers of the tapetum, corona radiata, and sagittal stratum. $\mathrm{Tp}=$ tapetum. Figure is available in color online only.

in all studied hemispheres. Furthermore, the white matter layers that were encountered en route to the roof of the ventricular atrium were categorized in discrete layers for educational purposes.

Thus, after the gray matter is removed, the first white matter layer identified consists of the arcuate or U fibers, which connect anatomofunctionally adjacent cortical areas (Fig. 1). Dissecting these fibers exposes the posterior 

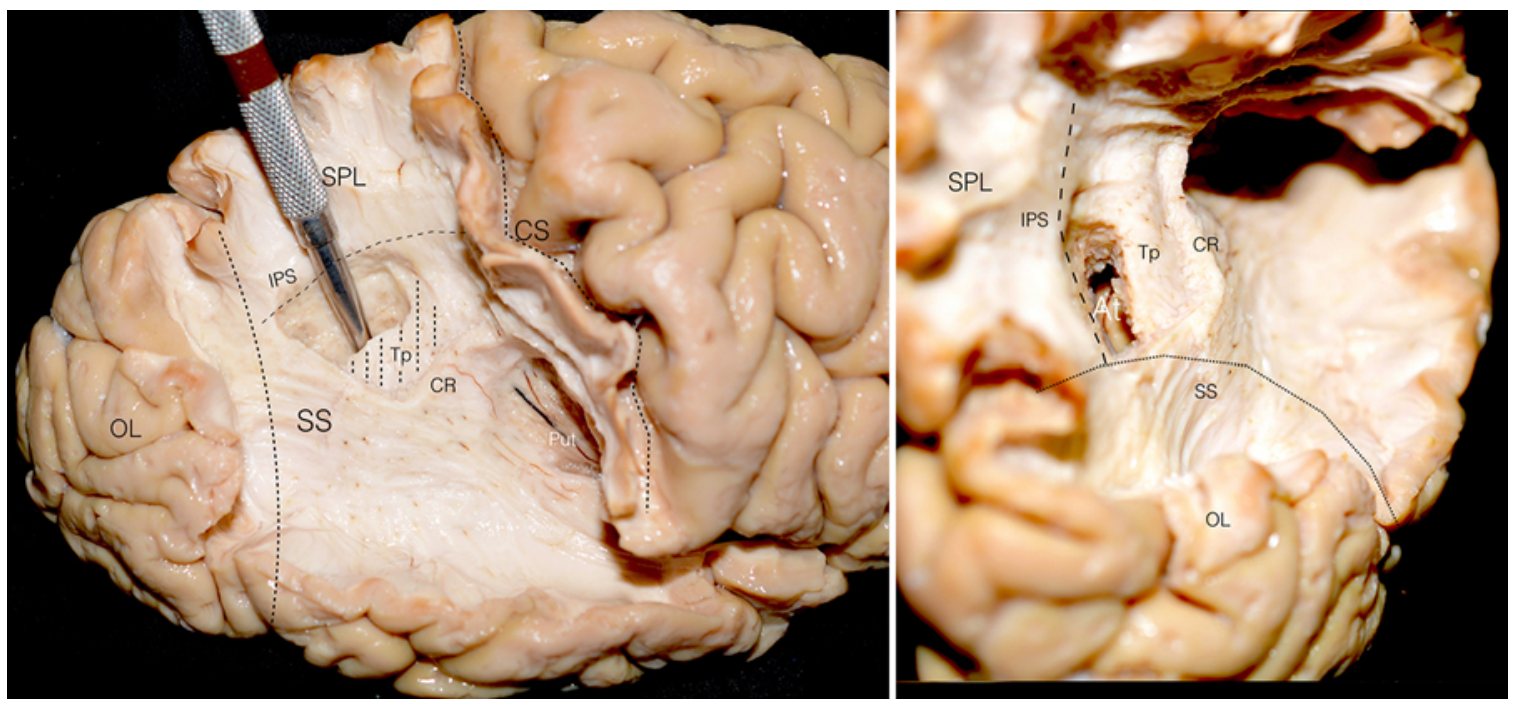

FIG. 6. Left: Photograph of the atrium entered by incising the layer of the tapetum. Note once again that the plane of the intraparietal sulcus corresponds to the roof of the atrium. Right: Oblique photograph of the same specimen. The atrium has been opened. The regional white matter anatomy can also be seen from a different angle. At = atrium. Figure is available in color online only.

part of the superior longitudinal fasciculus (SLF) and, more specifically, its horizontal, arcuate, and vertical segments, all shown in Fig. 2. Removing the SLF reveals the most laterally situated fibers of 2 compact bundles known as the corona radiata and sagittal stratum, which exhibit a continuity at the parietal and posterior temporal areas, respectively. At this level, these are claustrocortical radiation fibers forming the external capsule as they advance in the region of the insula and central core (Fig. 3).

During the next step, we had to extend the dissection to the posterior part of the central core and expose the gray matter of the putamen to differentiate the claustrocortical fibers from the fibers of the internal capsule in the region of interest. Thus, in Fig. 4 we show the posterior part of the putamen and the fibers of the posterior and retrolenticular limbs of the internal capsule, which merge at the periphery to form the corona radiata and sagittal stratum. We consistently observed that the fibers of the internal capsule that blend to form the sagittal stratum do not radiate above the plane defined by the intraparietal sulcus; therefore, the atrial roof is devoid of them. In contrast, the fibers that cover the roof of the atrium at this level radiate from the central core toward the superior parietal lobule, merging to form initially the corona radiata and subsequently the centrum semiovale; they are mainly parietal thalamic radiations and parietopontine fibers. No fibers covering the roof of the atrium were observed to bend toward the occipital lobe, hence mimicking the direction and giving the impression of the optic radiation. Because the optic radiation is entirely incorporated into the sagittal stratum and has the characteristic trajectory toward the occipital lobe and not to the superior parietal lobule, it becomes evident that the roof of the atrium is not lined by fibers of the visual pathway.

In the next step, we focused our dissection on the white matter overlying the atrial roof. By removing fibers of the corona radiata-centrum semiovale, we exposed the next and last layer en route to the ventricle consisting of the
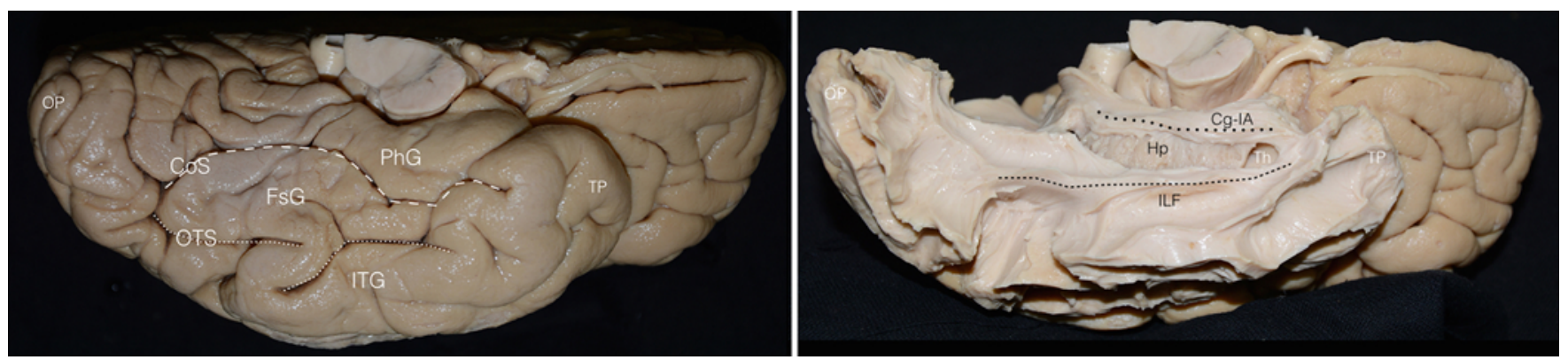

FIG. 7. Left: Photograph of the inferior cerebral surface of a left hemisphere. Dashed and dotted lines correspond to the collateral and occipitotemporal sulci, respectively. Right: Gradual dissection of the same specimen reveals the inferior longitudinal fasciculus (a fiber tract that runs at a level deep to the fusiform gyrus) and the inferior arm of the cingulum. The temporal horn has been entered, and the gray matter of the hippocampus is shown. $\mathrm{Cg}-\mathrm{IA}=$ inferior arm of the cingulum; $\mathrm{CoS}=$ collateral sulcus; $\mathrm{FsG}=$ fusiform gyrus; $\mathrm{Hp}=$ hippocampus; ILF = inferior longitudinal fasciculus; ITG = inferior temporal gyrus; OP = occipital pole; OTS = occipitotemporal sulcus; $\mathrm{PhG}=$ parahippocampal gyrus; Th = temporal horn; TP = temporal pole. Figure is available in color online only. 


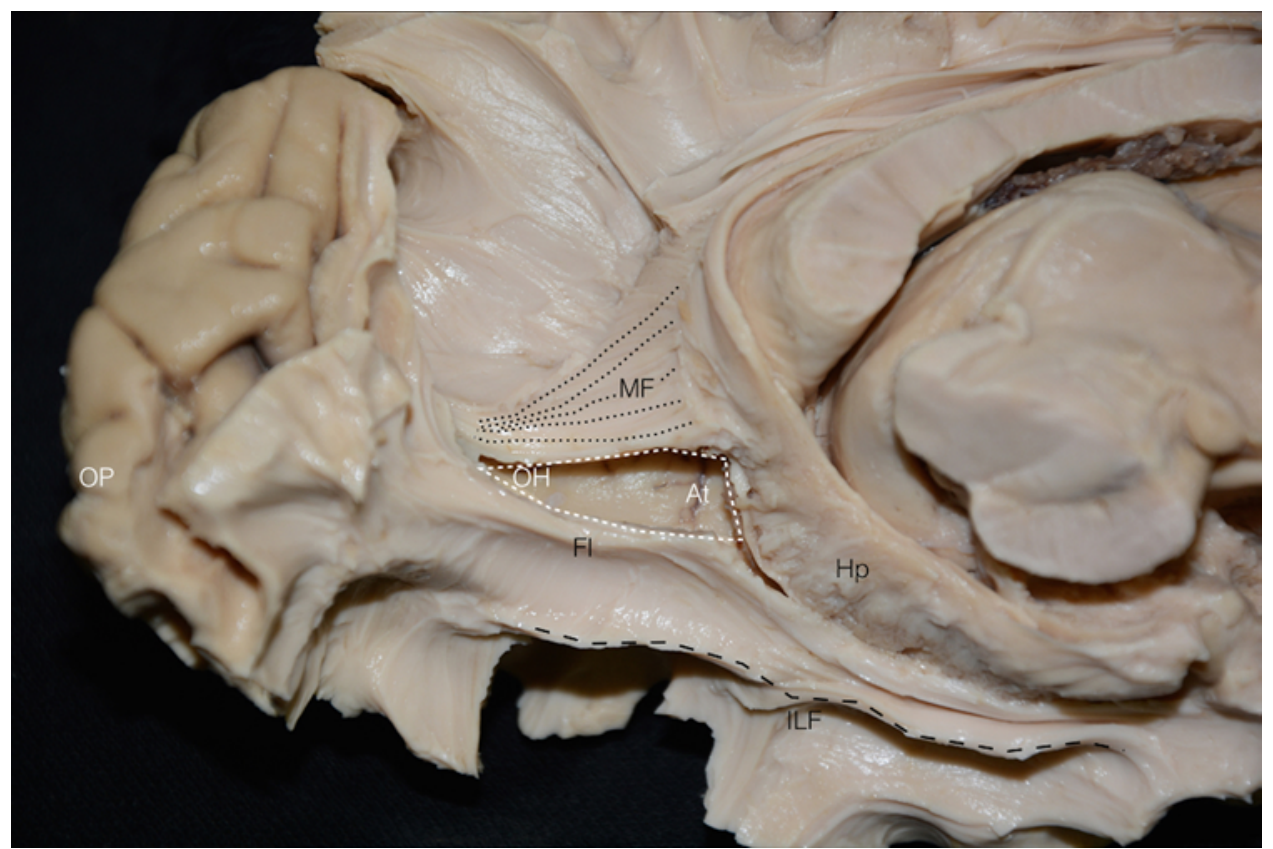

FIG. 8. Photograph of the medial aspect of the hemisphere after dissection of a part of the cingulum and forceps major. The atrium and occipital horn are entered through their medial wall, as shown. The floor of the temporal horn, atrium, and occipital horn is left intact. Note that the medial wall of the atrium, temporal horn, and occipital horn is devoid of optic radiation fibers. $\mathrm{FI}=\mathrm{floor}$ of atrium and occipital horn; MF = major forceps; $\mathrm{OH}=$ occipital horn. Figure is available in color online only.

tapetum (Fig. 5). The tapetal fibers arise from the splenium of the corpus callosum and arch over the roof and lateral wall of the atrium exhibiting a dorsocaudal direction. Incising these fibers enables entry to the roof of the ventricular atrium, as shown in Fig. 6.

\section{Corticosubcortical Correlation of the Intraparietal Sulcus Topography}

In all studied hemispheres, the trajectory of the intraparietal sulcus was consistently observed to correspond to the roof of the atrium. Thus, by keeping the direction of the dissection along the plane of the intraparietal sulcus, we always approached the atrium through its roof. When the subcortical dissection was directed inferior to the plane of the intraparietal sulcus and therefore within the boundaries of the inferior parietal lobule, the atrium was entered through its lateral wall. As a result, the plane of the intraparietal sulcus proved to demarcate not only the superior from the inferior parietal lobule but also the anatomical transition of the roof to the lateral wall of the ventricular trigone and thus serves as an important surface landmark for proper orientation.

In addition, we observed the superior extension of the parietal component of the SLF to lie adjacent to the plane of the intraparietal sulcus. This relation was particularly tight regarding the most anterior part of the intraparietal sulcus and the SLF, a finding also documented in a previous anatomical study. ${ }^{19}$

\section{Atrial Floor and Optic Radiations}

To delineate the relationship between the optic radiation and the floor of the atrium, we performed white mat- ter dissections focused on the mediobasal temporo-occipital cerebral surface, as mentioned in Methods.

Thus, by removing the gray matter of the parahippocampal gyrus, collateral sulcus, isthmus of the cingulum, fusiform, and inferior temporal and lingual gyri, the small arcuate (U) fibers of this cerebral area are exposed and comprise the first white matter layer identified. Dissection of these fibers reveals medially the inferior arm of the cingulum, extending from the isthmus of the cingulum to the parahippocampal gyrus, and laterally (deep to the fusiform gyrus) a straight and well-defined fiber bundle coursing from the temporal pole toward the occipital lobe known as the inferior longitudinal fasciculus. At this stage, the temporal horn has been entered through its floor and the body of the hippocampus is exposed, as shown in Fig. 7. By following the temporal horn to its gradual transition to the atrium and by tilting the direction of the dissection medially at the level of the medial occipital surface known as the cuneus, we expose the fibers of the forceps major radiating from the splenium of the corpus callosum over the medial wall of the atrium and occipital horn (Fig. 8). By removing a portion of the fibers of the forceps major along with the ventricular ependyma, we enter both the atrium and occipital horn through their medial wall, which is known to be devoid of optic radiations (Fig. 8). At this stage, we can properly identify the different ventricular compartments (i.e., temporal horn, atrium, and occipital horn) that reside in the mediobasal, temporooccipital region. During the last step of the dissection, we gradually remove the hippocampus, inferior longitudinal fasciculus, and ventricular ependyma and reveal the fibers of the tapetum, which arch over the lateral wall of the atrium, along 

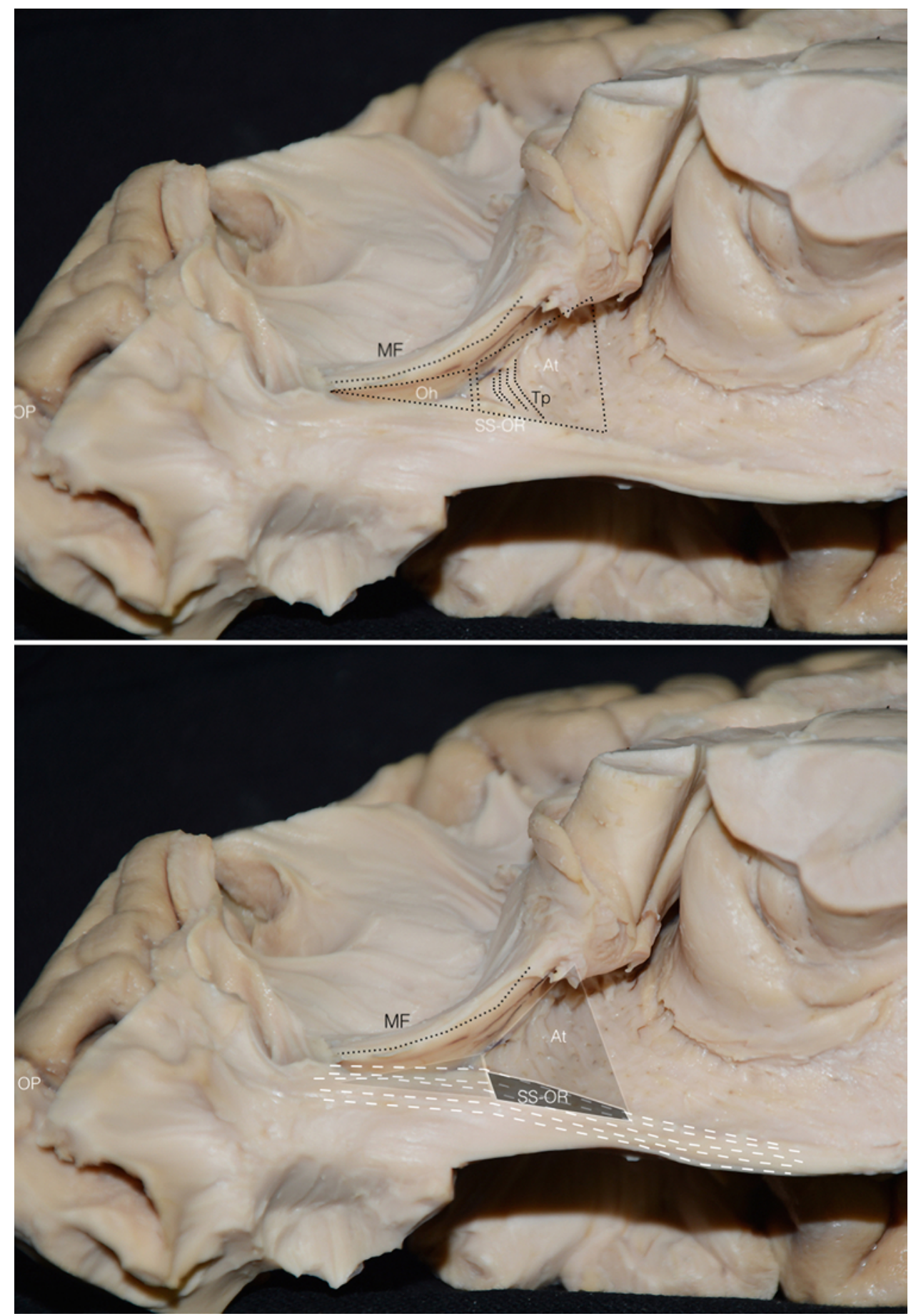

FIG. 9. Upper: Photograph of the inferior view of the specimen. Progressive dissection exposes the temporal horn, atrium, and occipital horn. The fibers of the tapetum are shown arching over the lateral wall of the atrium, along with the fibers of the sagittal stratum-optic radiations that sweep around to cover the inferior wall of the occipital horn, as shown in the photograph. The atrium and occipital horn are demarcated for better orientation. Lower: Magnified photograph of the floor of the atrium and occipital horn, clearly showing the topographic relationship between the optic radiation and the atrial floor. Note the fibers that course along the lateral part of the atrium that gradually bend to cover the entire floor of the occipital horn. These fibers have the characteristic course and trajectory of the anterior optic bundle. Oh = occipital horn; SS-OR = fibers of sagittal stratum-optic radiation. Figure is available in color online only.

with the fibers of the optic radiation-sagittal stratum that sweep around the lateral wall of the temporal horn (Fig. 9). While focusing on the floor of the atrium, we consis- tently observed fibers coursing along its outer part that gradually turn over to the basal surface and cover completely the floor of the occipital horn (Fig. 9). These fibers 

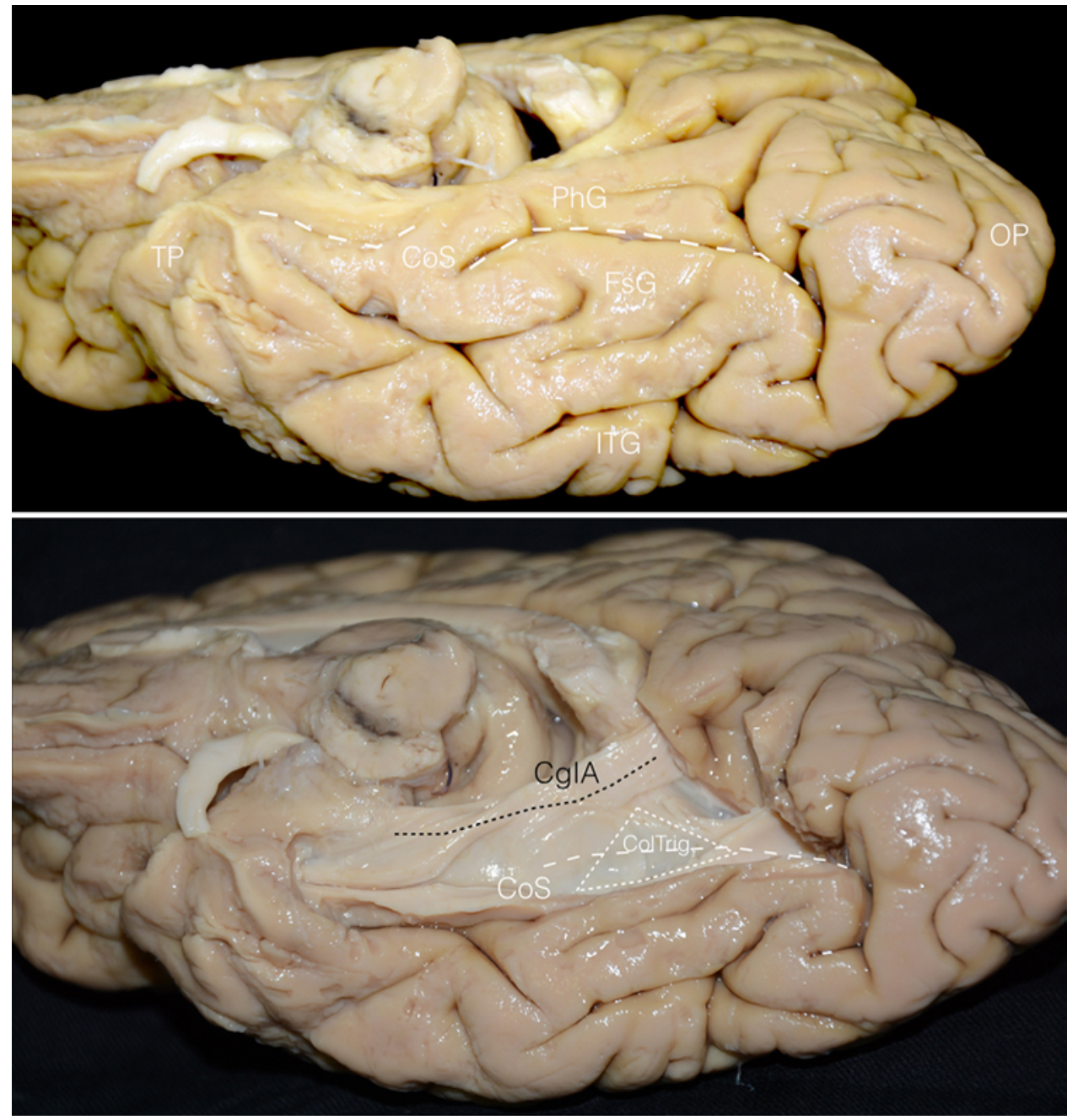

FIG. 10. Upper: Photograph of the inferior view of the right cerebral hemisphere. Surface anatomy is shown. The collateral sulcus is interrupted and marked with a dotted line. Lower: Focused dissection of the parahippocampal gyrus, collateral sulcus, and its adjacent cerebral territory reveals the inferior arm of the cingulum coursing medially and a subependymal white matter mantle investing most of the floor of the atrium, which extends up to the medial wall of the atrium and reaching the inferior arm of the cingulum. The anatomical silhouette of the atrium is marked for better orientation and understanding. ColTrig $=$ collateral trigone. Figure is available in color online only.

belong to the anterior optic bundle because they exhibit a characteristic course, trajectory, orientation, and location and were observed to occupy the outer/lateral part of the floor of the atrium in all studied hemispheres.

\section{Correlation Between the Collateral Sulcus Topography and Floor of the Atrium}

The collateral sulcus (i.e., the sulcus demarcating the parahippocampal and lingual gyri from the fusiform gyrus $^{23,40}$ ) was consistently observed to be a very deep sulcus, not only in its anterior temporal segment, in which it, as a result, forms a characteristic indentation to the floor of the temporal horn known as the collateral eminence, but in its entire length along the basal temporooccipital surface.

By focusing our dissection on the collateral sulcus in 5 specimens and by removing the gray matter and short arcuate fibers of the adjacent part of the fusiform and the parahippocampal gyri, we immediately encountered a thin subependymal white matter mantle investing most of the floor of the atrium (Fig. 10). This mantle was observed to extend to the medial wall of the atrium, up until it reaches the fibers of the inferior cingulum and major forceps. The most lateral/outer part of the atrial floor is covered by optic radiation fibers, as previously highlighted. Incising this white matter mantle enables entry to the atrium through its floor and medial wall, avoiding injury to the fibers of the optic pathway (Fig. 11).

However, we observed in all 5 hemispheres that the trajectory of the collateral sulcus at its posterior temporal part (i.e., the part that corresponds to the level of the ventricular atrium), when followed, was always pointing toward the lateral atrial floor, therefore presenting a risk for injury to the fibers of the optic radiation and sagittal stratum in general. Because the number of specimens studied by that mean was limited, we further investigated the relationship between the direction of the collateral sulcus and the atrial 


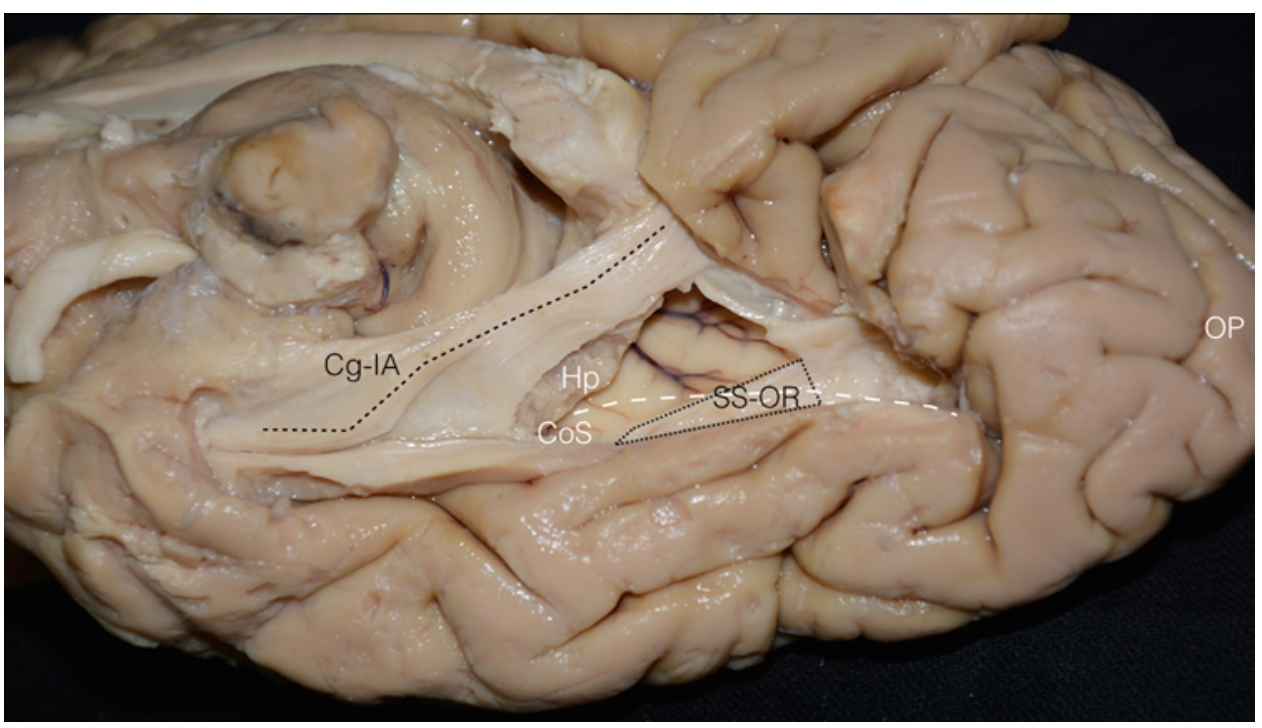

FIG. 11. Photograph of the same specimen as that shown in Fig. 10. Incising this subependymal white matter mantle enables entry into the posterior part of the temporal horn and atrium of the lateral ventricle. Note once again that the most lateral part of the floor of the atrium is covered by fibers of the optic radiation. Special emphasis should be placed on the trajectory of the collateral sulcus at its posterior temporal part, which in this specimen is seen to point toward the outer part of the atrial floor. Figure is available in color online only.

floor in 30 focused coronal MRI slices at the level of the atrium (60 collateral sulci). It was revealed that in 50\% (30 of 60 collateral sulci) of the hemispheres, the sulcus was pointing toward the lateral part of the atrial floor, in $38 \%$ (23 of 60 sulci), the sulcal trajectory was leading outside the ventricular trigone and into the temporal lobe's white matter, and in the remaining $12 \%$ (7 of 60 sulci), the collateral sulcus was observed to course toward the medial aspect of the atrial floor.

\section{Discussion}

Although previous laboratory studies have explored the microsurgical anatomy of the visual pathways and investigated their possible implications in the most commonly used approaches to the ventricular compartmen ts, $5,8,9,12,13,15,19,27,29,33,36,37,42-44$ evidence regarding the specific relationship of the optic radiations to the floor and roof of the ventricular trigone is ambiguous. Although some authors topographically related the atrial roof and floor to the fibers of the posterior and/or central optic bundle and anterior optic bundle, respectively, others did not define the precise anatomical correlation. ${ }^{5,9,15,27,30,36,37,42}$ In that respect, and by reviewing the relevant literature, we have listed in Table 1 the most recent and detailed papers on the optic pathway fiber tract architecture along with the authors' relevant comments on the relationship of the optic tract to the atrial floor and roof. In this table, it is evident

TABLE 1. Anatomical studies pertinent to the optic radiations and relevant comments on their relation to the atrial roof and floor

\begin{tabular}{llll}
\hline \multicolumn{1}{c}{ Authors \& Year } & \multicolumn{1}{c}{ Type of Study } & Relation of OR to the Roof & Relation of OR to the Floor \\
\hline Ebeling \& Reulen, 1988 & Anatomical/WMD & Yes/central bundle & No \\
\hline Rubino et al., 2005 & Anatomical/WMD & Not defined & Yes/anterior bundle \\
\hline Peltier et al., 2006 & Anatomical/WMD & Yes/central \& posterior & Not defined \\
\hline Kawashima et al., 2006 & Anatomical/WMD & Not defined & No \\
\hline Mahaney \& Abdulrauf, 2008 & Anatomical/WMD & Yes/posterior bundle & Yes/anterior bundle \\
\hline Izci et al., 2009 & Anatomical & Not defined & No \\
\hline Nayar et al., 2010 & Anatomical & Not defined & Not defined \\
\hline Martino et al., 2013 & Anatomical/WMD \& DTI & Not defined & Not defined \\
\hline Jeelani et al., 2017 & Anatomical & Not defined & No \\
\hline Párraga et al., 2012 & Anatomical/WMD & Yes/posterior bundle & Yes/anterior bundle \\
\hline Sampath et al., 2014 & Anatomical & Not defined & Not defined \\
\hline Güngör et al., $2017^{*}$ & Anatomical/WMD & No & Yes/anterior bundle \\
\hline
\end{tabular}

$\mathrm{DTI}=$ diffusion tensor imaging; $\mathrm{OR}=$ optic radiations; $\mathrm{WMD}=$ white matter dissection.

* With regard to the atrial floor, the only study that defined the anatomical correlation, stating that the optic radiations and, more specifically, the anterior optic bundle, courses on the lateral part of the atrial floor is that by Güngör et al. (2017). 
that the current neurosurgical literature is not only vague but at times conflicting with regard to this topic; therefore, studies that focus on the specific relationship of the optic radiation to the aforementioned parts of the ventricular trigone should be conducted.

In this context, one could argue that modern and sophisticated neuroimaging methods, such as diffusion tensor imaging and tractography, which can reconstruct and noninvasively map white matter cerebral anatomy in vivo, can be used to elucidate the complex 3D architecture of specific fiber tracts and provide sound morphological data. However, even with this recent advent, the accurate topographic anatomy of the optic radiation has yet to be clarified. . $, 8,11,24,26,35,48$ It should be noted that these elegant radiological methods, when applied to narrow and anatomically complex parenchymal areas, have the tendency to generate multiple artifacts as a result of the effect of "crossing,"

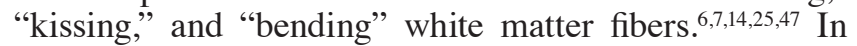
particular, and with regard to the optic radiations, which have a complex inherent trajectory and morphology, even the most advanced neuroimaging techniques are prone to anatomical inaccuracies, distortions, and errors and are not superior to the classical dissection methods. ${ }^{2,11,24,26,35,48}$ The fiber microdissection technique is still the gold standard for exploring intricate white matter pathways, which is why we chose it to be our basic method of investigation. $11,19,22,24,26,30,35,48$

Through these focused white matter dissections, we consistently observed the roof of the atrium to be covered by claustrocortical fibers, parietopontine fibers, and fibers of the parietal thalamic radiation and tapetum and found no evidence of optic radiation fibers arching over this segment of the ventricular trigone. Accordingly, neurosurgical approaches that aim to access the atrium through its roof do not potentially place the visual pathway at risk of injury and therefore do not increase surgery-related morbidity. Because the plane of the intraparietal sulcus was always seen to correspond to the roof of the atrium, ${ }^{10,17-19,40,41}$ operative trajectories directed through the superior parietal lobule and intraparietal sulcus are considered to be safe and effective corridors for treating intraatrial lesions. However, when the subcortical dissection is tilted inferior to the level of the intraparietal sulcus, within the boundaries of the inferior parietal lobule, then the atrium is entered through its lateral wall, the optic radiations are hit, which results in postoperative visual deficit.

With regard to the floor of the atrium, we consistently found fibers of the visual pathway coursing on its most lateral/outer part in all studied hemispheres. These fibers exhibit the characteristic direction, trajectory, orientation, and termination pattern of the anterior optic bundle as they bend inferiorly to cover completely the floor of the occipital horn. As a result, the surgeon should be very diligent when approaching the atrium through its floor and try to tilt the operative trajectory as medially as possible to avoid disrupting anterior optic bundle fibers lying in the lateral atrial floor, which can result in postoperative upper quadrant hemianopia. This knowledge is of great importance when it comes to presurgical planning and approach-related decision making for the treatment of patients harboring an atrial lesion with no evidence of preoperative visual deficit.
During our study, particular emphasis was placed on the correlative topography of the intraparietal and collateral sulci to the roof and floor of the ventricular atrium, respectively, because these sulci have been used as surgical corridors for treating atrial lesions because of their short and direct access to the ventricle. 1,9,10,12,13,15,17-19,29,41,50 In this context, the intraparietal sulcus, when it has the appropriate morphological characteristics (i.e., a straight, continuous sulcal segment of at least $3 \mathrm{~cm}$ for microsurgical dissection), offers a safe and effective trajectory to the atrium that avoids injury to the optic pathway. ${ }^{12,15,17-19,21,41}$ However, there is a tight relation between the plane of subcortical dissection achieved and the location of the SLF, particularly at the most anterior part of the sulcus. ${ }^{19}$ This relation is of particular surgical significance when approaching lesions on the dominant hemisphere, because the SLF is known to participate in the phonological and semantic pathways of language. . $^{3,4,28}$

In addition, the part of the collateral sulcus that corresponds to the ventricular trigone and is used during the transtentorial transcollateral sulcus approach was observed to point either toward the lateral part of the atrial floor or even outside the ventricle in $88 \%$ of the hemispheres we studied, therefore conferring risk of potential injury to optic radiation fibers and resulting in postoperative visual field deficit. Only in $12 \%$ of the hemispheres did the collateral sulcus, when followed, safely lead to the medial part of the floor of the atrium. Therefore, it is evident that a thorough preoperative assessment of the special morphological characteristics of the sulcus in terms of its length, depth, trajectory, branching pattern, and continuity in focused MRI scans, ${ }^{38}$ in combination with the proper incorporation of intraoperative neuronavigation, not only determines the feasibility of the approach in a given clinical case but also increases its safety and efficacy, which decreases surgery-related morbidity.

\section{Conclusions}

A focused fiber microdissection technique was used to clarify the topographic correlation of the atrial roof and floor to the fibers of the visual pathway, because it is considered the gold-standard method for exploring confined cerebral territories with intricate white matter architecture. Thus, in contrast to previous thought, the roof of the atrium proved to be devoid of optic radiation fibers, whereas the atrial floor was consistently observed to harbor optic fibers along its lateral, most outer part. Accordingly, operative trajectories that access the atrium should be evaluated with regard to their safety and efficacy and the preoperative clinical status of the patient. In that respect, the intraparietal and collateral sulci can be used as potential corridors to the ventricular trigone when particular morphological characteristics that determine the feasibility and safety of the relevant transventricular approach are adequately appreciated during meticulous presurgical planning.

\section{References}

1. Bertalanffy H, Krayenbuhl N, Wess C, Bozinov O: Ventricular tumors, in Winn HR (ed): Youmans Neurological Surgery, ed 6. Philadelphia: Elsevier, 2011, pp 1534-1568 
2. Borius PY, Roux FE, Valton L, Sol JC, Lotterie JA, Berry I: Can DTI fiber tracking of the optic radiations predict visual deficit after surgery? Clin Neurol Neurosurg 122:87-91, 2014

3. Catani M, Jones DK, ffytche DH: Perisylvian language networks of the human brain. Ann Neurol 57:8-16, 2005

4. Duffau H, Capelle L, Sichez N, Denvil D, Lopes M, Sichez JP, et al: Intraoperative mapping of the subcortical language pathways using direct stimulations. An anatomo-functional study. Brain 125:199-214, 2002

5. Ebeling U, Reulen HJ: Neurosurgical topography of the optic radiation in the temporal lobe. Acta Neurochir (Wien) 92:29-36, 1988

6. Fernandez-Miranda JC, Pathak S, Engh J, Jarbo K, Verstynen T, Yeh FC, et al: High-definition fiber tractography of the human brain: neuroanatomical validation and neurosurgical applications. Neurosurgery 71:430-453, 2012

7. Fernández-Miranda JC, Rhoton AL Jr, Alvarez-Linera J, Kakizawa Y, Choi C, de Oliveira EP: Three-dimensional microsurgical and tractographic anatomy of the white matter of the human brain. Neurosurgery 62 (6 Suppl 3):989-1028, 2008

8. Goga C, Türe U: The anatomy of Meyer's loop revisited: changing the anatomical paradigm of the temporal loop based on evidence from fiber microdissection. J Neurosurg 122:1253-1262, 2015

9. Güngör A, Baydin S, Middlebrooks EH, Tanriover N, Isler C, Rhoton AL Jr: The white matter tracts of the cerebrum in ventricular surgery and hydrocephalus. J Neurosurg 126:945-971, 2017

10. Harkey HL, al-Mefty O, Haines DE, Smith RR: The surgical anatomy of the cerebral sulci. Neurosurgery 24:651-654, 1989

11. Hofer S, Karaus A, Frahm J: Reconstruction and dissection of the entire human visual pathway using diffusion tensor MRI. Front Neuroanat 4:15, 2010

12. Izci Y, Seçkin H, Ateş O, Başkaya MK: Supracerebellar transtentorial transcollateral sulcus approach to the atrium of the lateral ventricle: microsurgical anatomy and surgical technique in cadaveric dissections. Surg Neurol 72:509-514, 2009

13. Jeelani Y, Gokoglu A, Anor T, Al-Mefty O, Cohen AR: Transtentorial transcollateral sulcus approach to the ventricular atrium: an endoscope-assisted anatomical study. J Neurosurg 126:1246-1252, 2017

14. Johansen-Berg H, Rushworth MF: Using diffusion imaging to study human connectional anatomy. Annu Rev Neurosci 32:75-94, 2009

15. Kawashima M, Li X, Rhoton AL Jr, Ulm AJ, Oka H, Fujii K: Surgical approaches to the atrium of the lateral ventricle: microsurgical anatomy. Surg Neurol 65:436-445, 2006

16. Klingler J, Ludwig E: Atlas cerebri humani. Basel: Karger, 1956

17. Koutsarnakis C, Kalyvas AV, Stranjalis G: Approaches to the ventricular atrium. J Neurosurg 126:1373-1374, 2017

18. Koutsarnakis C, Liakos F, Kalyvas AV, Komaitis S, Stranjalis G: White matter fiber tract architecture and ventricular surgery. J Neurosurg 126:1368-1371, 2017 (Letter)

19. Koutsarnakis C, Liakos F, Kalyvas AV, Liouta E, Emelifeonwu J, Kalamatianos T, et al: Approaching the atrium through the intraparietal sulcus: mapping the sulcal morphology and correlating the surgical corridor to underlying fiber tracts. Oper Neurosurg (Hagerstown) 13:503-516, 2017

20. Koutsarnakis C, Liakos F, Kalyvas AV, Sakas DE, Stranjalis G: A laboratory manual for stepwise cerebral white matter fiber dissection. World Neurosurg 84:483-493, 2015

21. Koutsarnakis C, Liakos F, Kalyvas AV, Skandalakis GP, Komaitis S, Christidi F, et al: The superior frontal transsulcal approach to the anterior ventricular system: exploring the sulcal and subcortical anatomy using anatomic dissections and DTI tractography. World Neurosurg 106:339-354, 2017

22. Koutsarnakis C, Liakos F, Liouta E, Themistoklis K, Sakas D, Stranjalis G: The cerebral isthmus: fiber tract anatomy, functional significance, and surgical considerations. J Neurosurg 124:450-462, 2016

23. Kubik S, Ono M, Abernathey CD: Atlas of the Cerebral Sulci. Stuttgart: Thieme, 1990

24. Kuhnt D, Bauer MH, Sommer J, Merhof D, Nimsky C: Optic radiation fiber tractography in glioma patients based on high angular resolution diffusion imaging with compressed sensing compared with diffusion tensor imaging-initial experience. PLoS One 8: 70973,2013

25. Le Bihan D, Poupon C, Amadon A, Lethimonnier F: Artifacts and pitfalls in diffusion MRI. J Magn Reson Imaging 24:478-488, 2006

26. Lim JC, Phal PM, Desmond PM, Nichols AD, Kokkinos C, Danesh-Meyer HV, et al: Probabilistic MRI tractography of the optic radiation using constrained spherical deconvolution: a feasibility study. PLoS One 10:e0118948, 2015

27. Mahaney KB, Abdulrauf SI: Anatomic relationship of the optic radiations to the atrium of the lateral ventricle: description of a novel entry point to the trigone. Neurosurgery 63 (4 Suppl 2):195-203, 2008

28. Makris N, Kennedy DN, McInerney S, Sorensen AG, Wang $\mathrm{R}$, Caviness VS Jr, et al: Segmentation of subcomponents within the superior longitudinal fascicle in humans: a quantitative, in vivo, DT-MRI study. Cereb Cortex 15:854-869, 2005

29. Marcus HJ, Sarkar H, Mindermann T, Reisch R: Keyhole supracerebellar transtentorial transcollateral sulcus approach to the lateral ventricle. Neurosurgery 73 (2 Suppl Operative):onsE295-onsE301, 2013

30. Martino J, da Silva-Freitas R, Caballero H, Marco de Lucas E, García-Porrero JA, Vázquez-Barquero A: Fiber dissection and diffusion tensor imaging tractography study of the temporoparietal fiber intersection area. Neurosurgery 72 (1 Suppl Operative):87-98, 2013

31. McDermott MW: Intraventricular meningiomas. Neurosurg Clin N Am 14:559-569, 2003

32. Nakamura M, Roser F, Bundschuh O, Vorkapic P, Samii M: Intraventricular meningiomas: a review of 16 cases with reference to the literature. Surg Neurol 59:491-504, 2003

33. Nayar VV, DeMonte F, Yoshor D, Blacklock JB, Sawaya R: Surgical approaches to meningiomas of the lateral ventricles. Clin Neurol Neurosurg 112:400-405, 2010

34. Nayar VV, Foroozan R, Weinberg JS, Yoshor D: Preservation of visual fields with the inferior temporal gyrus approach to the atrium. J Neurosurg 110:740-743, 2009

35. Nooij RP, Hoving EW, van Hulzen AL, Cornelissen FW, Renken RJ: Preservation of the optic radiations based on comparative analysis of diffusion tensor imaging tractography and anatomical dissection. Front Neuroanat 9:96, 2015

36. Párraga RG, Ribas GC, Welling LC, Alves RV, de Oliveira E: Microsurgical anatomy of the optic radiation and related fibers in 3-dimensional images. Neurosurgery 71 (1 Suppl Operative):160-172, 2012

37. Peltier J, Travers N, Destrieux C, Velut S: Optic radiations: a microsurgical anatomical study. J Neurosurg 105:294-300, 2006

38. Pruessner JC, Köhler S, Crane J, Pruessner M, Lord C, Byrne A, et al: Volumetry of temporopolar, perirhinal, entorhinal and parahippocampal cortex from high-resolution MR images: considering the variability of the collateral sulcus. Cereb Cortex 12:1342-1353, 2002

39. Rhoton AL Jr: The lateral and third ventricles. Neurosurgery 51 (4 Suppl):S207-S271, 2002

40. Ribas GC: The cerebral sulci and gyri. Neurosurg Focus 28(2):E2, 2010 
41. Ribas GC, Yasuda A, Ribas EC, Nishikuni K, Rodrigues AJ Jr: Surgical anatomy of microneurosurgical sulcal key points. Neurosurgery 59 (4 Suppl 2):ONS177-ONS211, 2006

42. Rubino PA, Rhoton AL Jr, Tong X, de Oliveira E: Three-dimensional relationships of the optic radiation. Neurosurgery 57 (4 Suppl):219-227, 2005

43. Sampath R, Katira K, Shi R, Vannemreddy P, Patil S, Nanda A: Radio-anatomic measurements and statistical generation of a safe surgical corridor to enter the ventricular trigone while avoiding injury to the optic radiations. J Neurol Surg A Cent Eur Neurosurg 75:453-461, 2014

44. Sarubbo S, De Benedictis A, Milani P, Paradiso B, Barbareschi M, Rozzanigo U, et al: The course and the anatomo-functional relationships of the optic radiation: a combined study with 'post mortem' dissections and 'in vivo' direct electrical mapping. J Anat 226:47-59, 2015

45. Sincoff EH, Tan Y, Abdulrauf SI: White matter fiber dissection of the optic radiations of the temporal lobe and implications for surgical approaches to the temporal horn. J Neurosurg 101:739-746, 2004

46. Türe U, Yaşargil MG, Friedman AH, Al-Mefty O: Fiber dissection technique: lateral aspect of the brain. Neurosurgery 47:417-427, 2000

47. Wang F, Sun T, Li XG, Liu NJ: Diffusion tensor tractography of the temporal stem on the inferior limiting sulcus. J Neurosurg 108:775-781, 2008

48. Wu W, Rigolo L, O’Donnell LJ, Norton I, Shriver S, Golby AJ: Visual pathway study using in vivo diffusion tensor im- aging tractography to complement classic anatomy. Neurosurgery 70 (1 Suppl Operative):145-156, 2012

49. Yaşargil MG: Microneurosurgery. Stuttgart: Thieme, 1984, Vol 1

50. Yaşargil MG, Abdulrauf SI: Surgery of intraventricular tumors. Neurosurgery 62 (6 Suppl 3):1029-1041, 2008

\section{Disclosures}

The authors report no conflict of interest concerning the materials or methods used in this study or the findings specified in this paper.

\section{Author Contributions}

Conception and design: Koutsarnakis. Acquisition of data: Koutsarnakis, Kalyvas, Komaitis, Liakos, Skandalakis. Analysis and interpretation of data: Koutsarnakis, Kalyvas, Komaitis, Skandalakis. Drafting the article: Koutsarnakis, Kalyvas, Komaitis, Liakos, Skandalakis. Critically revising the article: Koutsarnakis, Kalyvas, Stranjalis. Reviewed submitted version of manuscript: all authors. Approved the final version of the manuscript on behalf of all authors: Koutsarnakis. Administrative/technical/ material support: Anagnostopoulos, Stranjalis. Study supervision: Koutsarnakis.

\section{Correspondence}

Christos Koutsarnakis: Evangelismos Hospital, University of Athens, Greece.ckouts@hotmail.co.uk. 